CERN-TH/95-218

\title{
Reconnecting Coloured Dipoles
}

\author{
Leif Lönnblad
}

Theory Division, CERN

CH-1211 Genève 23, Switzerland

E-mail: Leif.Lonnblad@cern.ch

\begin{abstract}
Reconnections among the produced colour dipoles in the Dipole Cascade Model are discussed, and an implementation in the ARIADNE program is presented. The reconnections are shown to have only small effects on event shapes at LEP 1 , on the $W$ mass determination at LEP 2 and on rapidity gaps in deep inelastic scattering at HERA.
\end{abstract}

Submitted to Z. Phys. C

CERN-TH/95-218

August 1995 


\section{Introduction}

The effects of colour reconnections in multiparton final states has received some attention lately, not least because of the plans for measuring the $W$ mass at LEP 2. Early work [1] indicated that such effects could be quite large in $e^{+} e^{-} \rightarrow W^{+} W^{-} \rightarrow$ jets, when the quark from one $W$ decay forms a colour singlet with the antiquark of the other. However, later work $[2,3]$ showed that the effects of reconnections are very small on the perturbative level due to the finite lifetime of the $W$, while effects on the hadronization level still could affect the measurement of the $W$ mass to a small extent at LEP 2.

Recently, there have also been attempts to explain the sizeable fractions of events with a large rapidity gap in deep inelastic ep scattering (DIS) found at HERA $[4,5]$ in terms of colour reconnections [6-8]. The idea here is that the $q \bar{q}$ pair formed in $\gamma^{\star} g \rightarrow q \bar{q}$ events would form a colour singlet due to colour reconnections, and a rapidity gap would be formed between this $q \bar{q}$ pair and the proton remnant.

In this paper, a model for introducing colour reconnections in the Dipole Cascade Model $[9,10]$ is presented together with its implementation in the ARIADNE [11] event generator program. The model follows closely the one suggested in ref. [12], but differs in the details of the implementation, and in that it allows for more general studies of colour-reconnection effects.

The model and its implementation are described in section 2, and studies of the resulting effects on event shapes and multiplicities at LEP 1 , on the $W$ mass determination at LEP 2 and on DIS events at HERA are presented in sections 3, 4 and 5, respectively. Finally some conclusions are presented in section 6 .

\section{The model}

The Dipole Cascade Model (DCM) for $e^{+} e^{-}$annihilation and DIS is described in detail in refs. $[9,10,13]$ and only a brief summary of the features that are important for this paper will be given here.

The emission of a gluon $g_{1}$ from a $q \bar{q}$ pair created in an $e^{+} e^{-}$annihilation event can be described as radiation from the colour dipole between the $q$ and $\bar{q}$. A subsequent emission of a softer gluon $g_{2}$ can be described as radiation from two independent colour dipoles, one between the $q$ and $g_{1}$ and one between $g_{1}$ and $\bar{q}$, neglecting the contribution from the $q \bar{q}$ dipole, which is suppressed by $1 / N_{c}^{2}$. Further gluon emissions are given by three independent dipoles etc. In this way, the end result is a chain of dipoles, where one dipole connects two partons, and a gluon connects two dipoles. This is in close correspondence with the Lund string picture, where gluons act as kinks on a string-like field stretched 

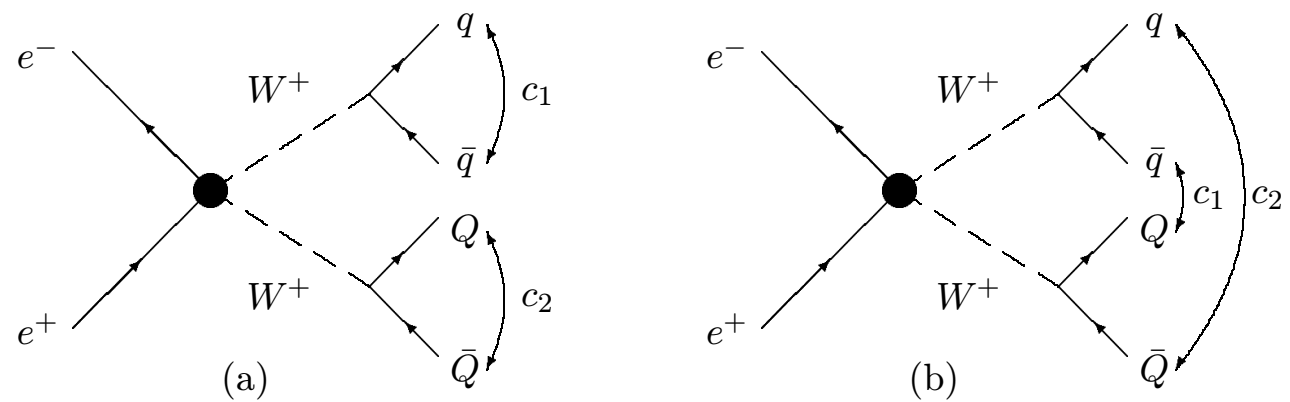

Figure 1: The different colour connection possibilities in $e^{+} e^{-} \rightarrow W^{+} W^{-} \rightarrow q \bar{q} Q \bar{Q}$ events.

between the $q \bar{q}$ pair.

In DIS, the gluon emission comes from the dipole stretched between the quark, struck by the electroweak probe, and the hadron remnant. The situation is the same as in $e^{+} e^{-}$above, except that, while $q$ and $\bar{q}$ are both point-like in the case of $e^{+} e^{-}$, the hadron remnant in DIS is an extended object. This results in an extra suppression of radiation in the remnant direction.

The potentially large effects of colour reconnections are perhaps most easily demonstrated in the case of $e^{+} e^{-} \rightarrow W^{+} W^{-} \rightarrow$ jets events. Here, there is a probability of $1 / N_{c}^{2}$ that the quark from one $W$ decay could form a colour singlet with the antiquark from the other as in fig. 1b. In the DCM, the connected quark-antiquark pairs would then form colour dipoles, which independently radiate gluons, and in the end the two systems would hadronize, again independently. It is clear that the two possibilities of colour connections in fig. 1 would give rise to very different radiation patterns for the gluons and therefore also very different flows of hadrons in the final state. The effects of the $W$ lifetime mentioned in the introduction will be discussed below.

The strategy of the implementation of colour reconnections in the DCM is to assign a colour index $c_{i}$ to each dipole $i$. The index is just a number chosen randomly in the interval $[1,9]$. If and only if two dipoles have the same colour index, they may be allowed to reconnect, so that the colour end of one dipole forms a new dipole with the anticolour end of the other and vice versa. It is clear that this procedure works in the $e^{+} e^{-} \rightarrow W^{+} W^{-} \rightarrow$ jets case in fig. 1 , but some care has to be taken to avoid unphysical colour flows.

First of all no adjacent dipoles can have identical colour indices. Otherwise the colour side of the connecting gluon would be allowed to form a dipole with the anticolour side of itself, resulting in a colour singlet gluon. Similarly, in fig. 2 only in diagram (a) should the two gluons be allowed to reconnect to form a colour singlet system, since in diagram (b) they come from the same colour octet gluon.

In the DCM, however, the two diagrams in fig. 2 are treated coherently, and they have do 

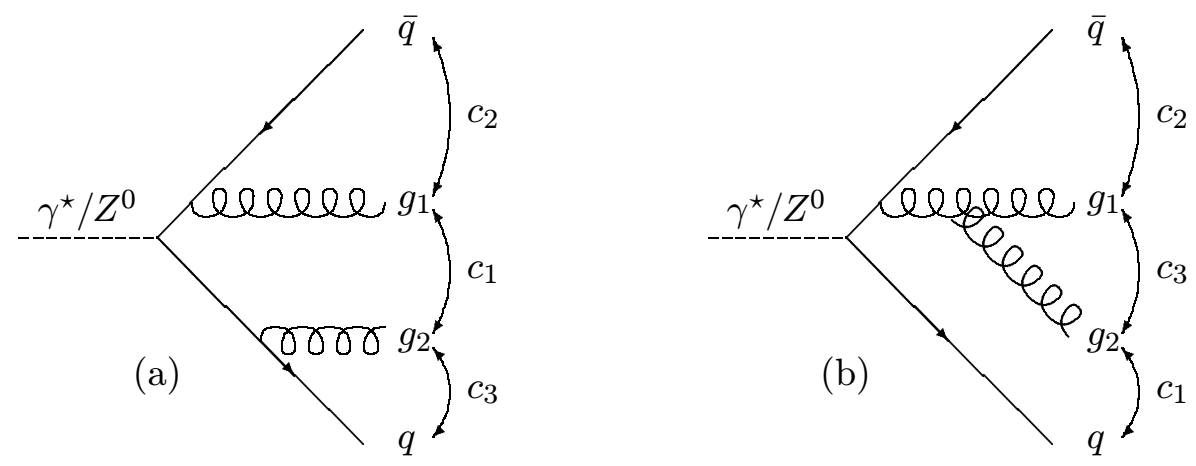

Figure 2: The colour connections after two gluon emissions in $e^{+} e^{-}$annihilation. In (a), $c_{2} \neq c_{1} \neq c_{3}$, while in (b) all colour indices are different.

be divided up by hand afterwards. The chosen procedure is to assign the emitted gluon to one of the emitting partons according to the same scheme as that used for deciding which of the emitting partons takes the transverse recoil in an emission [10,14]. If $x_{1}$ and $x_{3}$ are the final state energy fractions of the original partons, the gluon is thus assumed to have been radiated from parton 3 with the probability

$$
P_{3}\left(x_{1}, x_{3}\right)=\frac{x_{1}^{2}}{x_{1}^{2}+x_{3}^{2}},
$$

and vice versa.

The fact that two dipoles may reconnect from colour considerations does not mean that they must do so. Here the model of ref. [12] will be used, where only such reconnections will be performed, which minimizes the so-called lambda measure $[15,16]$. This is a generalized rapidity measure, corresponding to the "length" of a Lund string, defined as

$$
\lambda=\sum_{1}^{n-1} \ln \left(p_{i}+p_{i+1}\right)^{2} / m_{0}^{2}
$$

for a string with $n$ colour-ordered partons with four-momenta $p_{i} ; m_{0}$ is a hadronic mass scale around $1 \mathrm{GeV}$. The effect is that partons close in momentum space are more likely to be colour connected, which is a sensible assumption.

The full colour-reconnection procedure now looks as follows:

1 After each gluon radiation from a dipole with colour index $c_{1}$, the dipole between this gluon and the parton from which it is said to have been emitted according to eq. (1), is given a new colour index $c_{2}$ different from $c_{1}$ and, if the emitting parton was a gluon, different from the index of the neighbouring dipole. The dipole between the radiated gluon and the other parton retains the index of the original dipole $c_{1}$. 
2 For a gluon that is split into a $q \bar{q}$ pair [17], the two connecting dipoles retain their colour indices.

3 After each emission, all pairs of dipoles with identical colour indices for which a reconnection would give a decreasing $\lambda$ are considered, and the one that gives the largest decrease in $\lambda$ is allowed to reconnect. This is repeated until no reconnection giving decreasing $\lambda$ is possible.

4 The dipole cascade is then allowed to continue using the possibly reconnected dipoles, with the reconnection procedure repeated in each emission.

With this procedure implemented in the ARIADNE event generator program, it is now possible to get some realistic estimates of the effects due to colour reconnections on the perturbative level in parton showers.

In principle this model could also be used together with the string reconnection scenario in refs. $[2,3]$, but although that model only looks at the non-perturbative stage, the model presented here also reconnects strings just after the perturbative cascade, which means that the two overlap and, in the following, the standard string fragmentation as implemented in JETSET $[18,19]$, without reconnections, is used.

\section{Event Shapes at LEP 1}

At LEP, the effects of colour reconnections should be small. First of all, the effects are suppressed by $1 / N_{c}^{2}$. But also, it can only affect emissions softer than the two hardest gluons, suppressing the effects with $\alpha_{S}^{2}$. In addition, the concept of pre-confinement says that colour-connected partons usually are close in phase space which, in the scenario presented here, means that for a given possible reconnection the original colour flow is usually favoured.

On the other hand, the measurements of event shapes and particle spectra at LEP have reached very high precision, and the standard parton-shower-based generators, such as HeRwig [20], JETSET and also ARIADne ${ }^{1}$, are known to fit these data extremely well (see e.g. ref. [21]). Therefore it is a reasonable requirement that any change in the underlying models should not change the description of these LEP results.

The mean charged multiplicity is one example of a precisely measured quantity at LEP. It is clear that introducing colour reconnections in ARIADNE as described above will decrease the multiplicity in that model, since all changes have the effect of decreasing the string lengths.

\footnotetext{
${ }^{1}$ Note that ARIADNE only generates the perturbative QCD cascade in an event and, in this paper, when events are said to be generated with ARIADNE, the hadronization is performed with JETSET and, where applicable, the hard sub-processes are generated with PYTHIA or LEPTO.
} 


\begin{tabular}{|c|c|}
\hline Model & $\left\langle\mathrm{N}_{\mathrm{ch}}\right\rangle$ \\
\hline Default & 21.20 \\
With reconnections & 20.84 \\
With reconnections and retuning & 21.16 \\
\hline
\end{tabular}

Table 1: The mean charged multiplicity in hadronic $Z^{0}$ decays at LEP according to the ARIADNE event generator. The statistical error in the simulation is $\approx 0.02$.

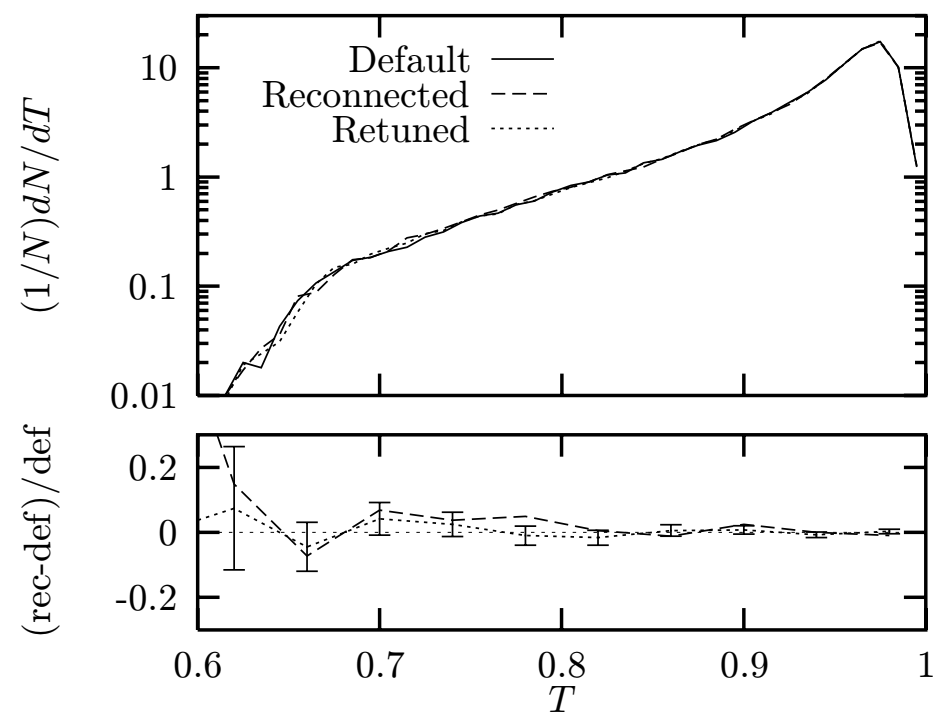

Figure 3: The thrust distribution in hadronic $Z^{0}$ events at LEP. Full line is ARIADNE with default settings, dashed line is the same with colour reconnections included and the dotted line is with colour reconnections and retuning. The upper plot is the actual distribution, while the lower shows the relative differences w.r.t. the default ARIADNE. The error bars in the lower plot correspond to the statistical error in the simulation.

To compensate for this, it is of course possible to change the parameters of the string fragmentation in JETSET and retain a good description of the LEP results. Ideally, such a retuning should be done simultaneously for many measured distributions, as in ref. [21]. However, that is a very complicated procedure, requiring a large amount of computing power, and only a crude such tuning will be presented here.

Figures 3, 4 and 5 and table 1 shows the ARIADNE description of some event characteristics at LEP. It is clear that the introduction of colour reconnections does affect most of these characteristics, but that the differences almost vanish already after a crude retuning. The $p_{\perp \text { out }}$ distribution in fig. 5 is an example of a distribution where this crude retuning failed to reduce the differences. It is interesting to note that for this distribution, the ARIADNE program does not describe LEP data very well [21], and the hardening introduced by the 


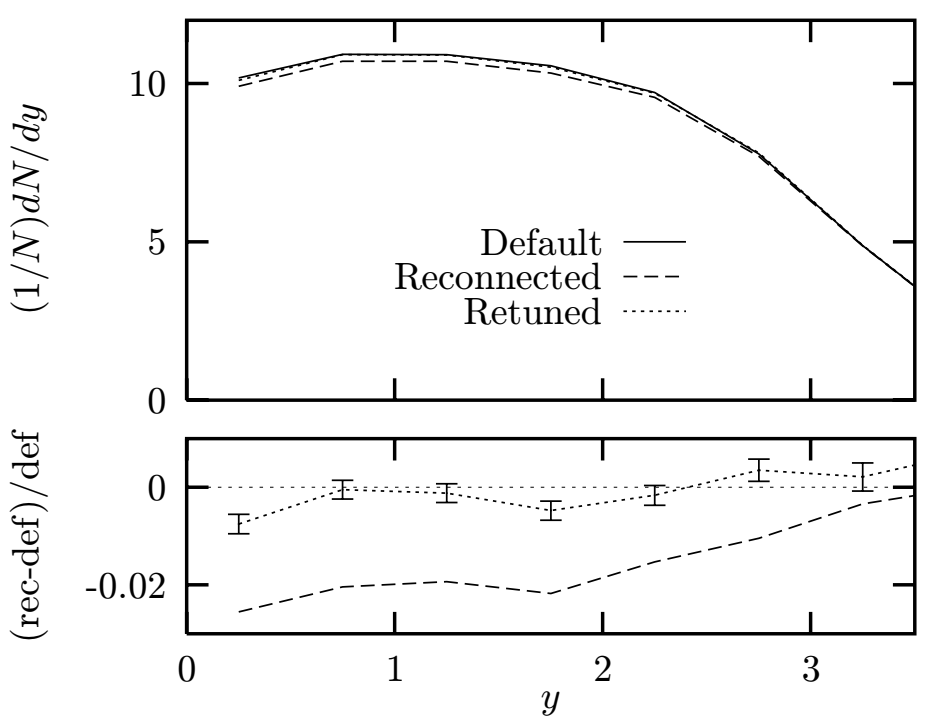

Figure 4: The rapidity distribution along the thrust axis of all particles in hadronic $Z^{0}$ events at LEP using the same notation as in fig. 3.

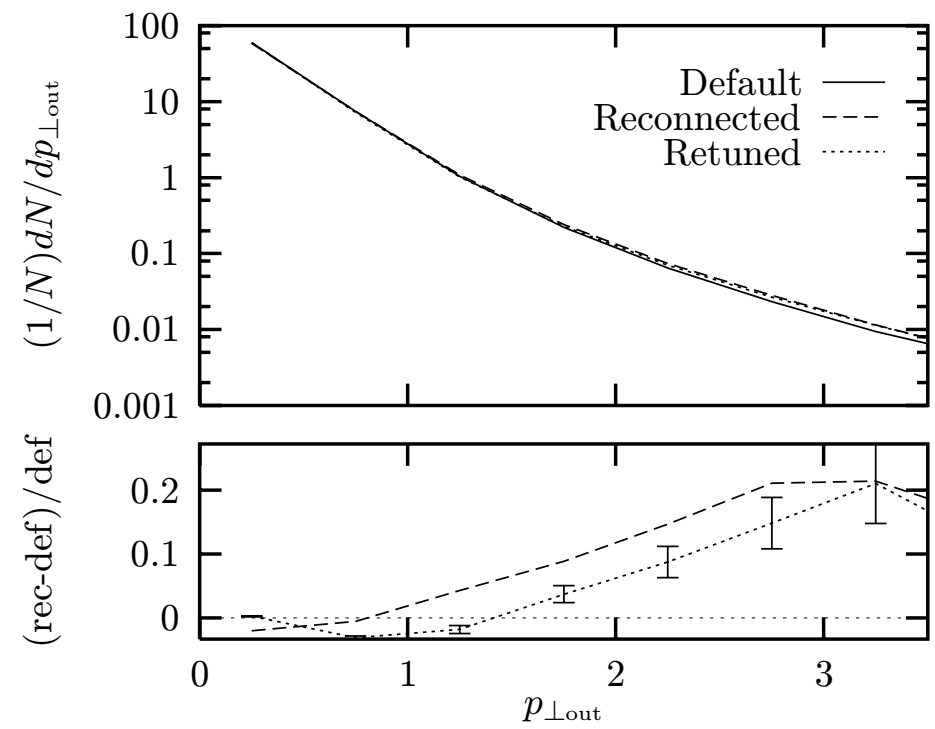

Figure 5: The $p_{\perp \text { out }}$ distribution of all particles in hadronic $Z^{0}$ events at LEP using the same notation as in fig. 3. $p_{\perp \text { out }}$ is defined as the momentum component perpendicular to the event plane defined in the thrust analysis. 
colour reconnections is favoured by data. However, no firm conclusions can be drawn before a more detailed retuning has been done.

\section{W mass determination at LEP 2}

At LEP 2, an important task is to precisely measure the mass of the $W$. One of the methods suggested for doing this is to look at the case where both the $W$ 's decay hadronically. By reconstructing jets and assigning them to the different $W$ 's, the mass of each $W$ can be measured on an event-by-event basis. It is clear that any "cross-talk" between the decay products of the individual $W$, such as colour reconnections on the perturbative or nonperturbative level, or even Bose-Einstein effects among the final state hadrons [22], could introduce a smearing or a systematical shift in the $W$ mass measurement.

In refs. $[2,3]$ it was shown that colour reconnections between the $W^{+}$and $W^{-}$on the perturbative level in $e^{+} e^{-} \rightarrow W^{+} W^{-} \rightarrow$ jets events are heavily suppressed due to the finite lifetime of $W$. In particular it was shown that gluons with energy $E_{g}>\Gamma_{W} \approx 2 \mathrm{GeV}$ are radiated incoherently by the two initial dipoles $q \bar{q}$ and $Q \bar{Q}$. Gluons emitted with smaller energy can in general be emitted coherently, but those with such small energy do not give large effects on the hadronic final state. Instead a model for non-perturbative reconnections among strings in the Lund string-fragmentation model was investigated, and the uncertainties in the measured $W$ mass introduced by such effects was estimated to approximately $30 \mathrm{MeV}$.

An investigation of the effects of colour reconnections between the two $W$ 's within the framework of the DCM was presented in ref. [12]. It was, however, limited to one reconnection per event, and did not take into account reconnections within each $W$. Using the model presented here, it is possible to make a more complete study of such reconnection effects.

The strategy will be to let the two dipole systems $q \bar{q}$ and $Q \bar{Q}$ evolve separately, radiating all possible gluons with $E_{g}>\Gamma_{W}$ independently and allowing colour reconnections only within each dipole system. After all such emissions are performed, reconnections between the two systems are switched on, and possible gluons with $E_{g}<\Gamma_{W}$ are radiated. Since the ordering in the DCM is in transverse momentum, the whole cascade is run twice from the maximum allowed $p_{\perp}$, down to the cutoff; first without cross-talk between the two systems, and allowing only gluons with $E_{g}>\Gamma_{W}$, and then again with cross-talk, allowing only $E_{g}<\Gamma_{W}$. This scheme is somewhat inconsistent as some high- $p_{\perp}$ gluons can be emitted after one with a lower $p_{\perp}$, contrary to what is assumed in the DCM, and as an alternative the second cascade can be omitted, but allowing for reconnections between the systems before fragmentation.

The effect on a $W$ mass measurement at LEP 2 is very dependent on the actual method 


\begin{tabular}{|l|c|c|}
\hline Model & $\begin{array}{c}\left\langle\Delta \bar{m}_{W}\right\rangle \\
(\mathrm{MeV})\end{array}$ & $\begin{array}{c}\left\langle\delta \bar{m}_{W}\right\rangle \\
(\mathrm{MeV})\end{array}$ \\
\hline (a) No reconnections & 98 & - \\
(b) Reconnections within each $W$ & 103 & 5 \\
(c) As (b) but retuned & 97 & -1 \\
(d) As (c) but reconnections between for $E_{g}<2 \mathrm{GeV}$ & 130 & 32 \\
(e) As (d) but no gluons with $E_{g}<2$ & 121 & 23 \\
(f) As (d) but for $E_{g}<5 \mathrm{GeV}$ & 156 & 58 \\
(g) No reconnections $E_{\mathrm{CM}}=180 \mathrm{GeV}$ & 140 & - \\
(h) As (d) but $E_{\mathrm{CM}}=180 \mathrm{GeV}$ & 190 & 50 \\
\hline
\end{tabular}

Table 2: The average difference $\left\langle\Delta \bar{m}_{W}\right\rangle$ between the reconstructed and generated $W$ mass, and the difference $\left\langle\delta \bar{m}_{W}\right\rangle=\left\langle\Delta \bar{m}_{W}\right\rangle^{\text {recon }}-\left\langle\Delta \bar{m}_{W}\right\rangle^{\text {no-recon }}$ w.r.t. the no-recoupling scenario using different models for the colour reconnections. The simulation was made at a centerof-mass energy of $170 \mathrm{GeV}$ except in $(\mathrm{g})$ and $(\mathrm{h})$. The statistical errors in the simulations are approximately $6 \mathrm{MeV}$ for $\left\langle\Delta \bar{m}_{W}\right\rangle$ and $8 \mathrm{MeV}$ for $\left\langle\delta \bar{m}_{W}\right\rangle$.

used for reconstructing the mass. To get an estimate here, a method similar to the one labelled 1 in ref. [3], for selecting events, reconstructing jets, and assigning them to the different $W$ 's was used. This is not an experimentally feasible method since, to assign the jets, it uses information about the initial quark directions from the generator. In each event the difference $\Delta \bar{m}_{W}$ between the average of the two reconstructed $W$ masses and the average of the two generated ones is measured. The result is presented in table 2.

Approximately $10^{5}$ events were generated for each model, which is more than is expected at LEP 2. Since the effects of reconnections are not far above the statistical error expected at LEP 2, it is clear that such effects are not large. Looking at the realistic scenarios (d), (e) and (g), the reconnections give a shift of about $30-50 \mathrm{MeV}$, which is consistent with the findings of ref. [3]. From table 2 it is also clear that the retuning introduced in the previous section gives a very small effect. Also, whether or not the cascade is continued below $E_{g}=\Gamma_{W}$ makes little difference. The procedure is, however, sensitive to the exact value of $E_{g}$ below which reconnections between the two $W$ systems are allowed, and to the center of mass energy used.

Again it should be stressed that the effects of colour reconnections depend heavily on the details of the method used to reconstruct the $W$ mass, and more realistic reconstructions will most likely smear out these effects. However, now that both the model presented here and the non-pertubative reconnection model in ref. [3] are available in ARIADNE and JETSET respectively, the study of effects on any method of $W$ mass reconstruction can be more easily estimated. 


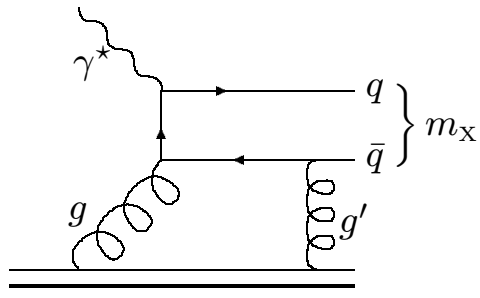

(a)

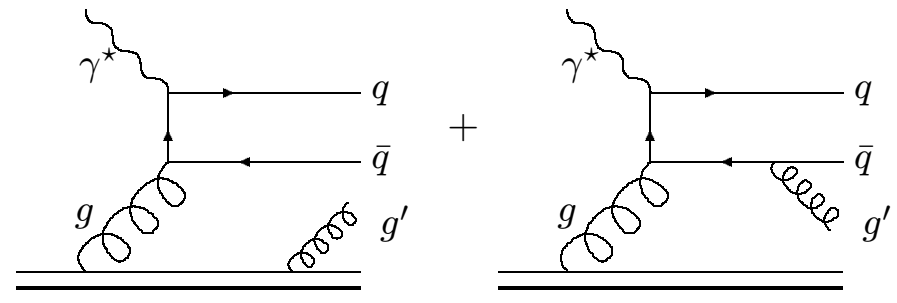

(b)

Figure 6: The $\gamma^{\star} g \rightarrow q \bar{q}$ subprocess in DIS. (a) describes the exchange of a soft gluon $g^{\prime}$, possibly leaving the $q \bar{q}$ pair in a colour-singlet state. (b) shows the two diagrams contributing to a gluon $g^{\prime}$, radiated from the dipole between the $\bar{q}$ and one part of the proton remnant.

\section{Rapidity gaps at HERA}

In ref. [23] a model was presented for describing the large rapidity-gap events in DIS found at HERA $[4,5]$ within the framework of the DCM. This model assumed that such events correspond to scattering of partons in a colourless sub-object within the proton, and was very similar to the pomeron model of Ingelman and Schlein [24].

Recently there have been attempts to explain the rapidity-gap events without the introduction of the pomeron, using instead colour-reconnection arguments. One such model [7] looks only at the $\mathcal{O}\left(\alpha_{S}\right)$ boson-gluon fusion (BGF) process, and assumes that the formed $q \bar{q}$ pair can exchange soft gluons with the proton remnant, as in fig. 6 a. In the following this model will be referred to as the Leading Order Reconnection (LOR) model. With a probability of $\approx 1 / N_{c}^{2}$, the $q \bar{q}$ pair is left in a colour singlet state after one or many such exchanges, and in that case the $q \bar{q}$ pair would hadronize independently from the proton remnant, leaving a large gap in rapidity without hadrons. In ref. [7] it was found that the LOR model could describe the rate of rapidity-gap events as well as the distribution of the mass $m_{\mathrm{X}}$ of the struck system.

However, the soft gluon in fig. 6a is exchanged very "late" in the process and can therefore not prevent any extra perturbative gluons from being radiated. And it is clear that any such gluon radiation, also very soft emissions, e.g. from the incoming gluon $g$, could easily destroy the rapidity gap between the $q \bar{q}$ pair and the proton remnant, and also alter the $m_{\mathrm{x}}$ distribution. In other words, the LOR model relies on the exchange diagram of fig. 6a, which may create rapidity gaps, but disregards completely the equivalent diagrams in fig. $6 \mathrm{~b}$, which may destroy the gaps again.

For the model to be meaningful, it is therefore important to also include higher order corrections, preferably using an "all order" scenario such as the parton cascade approach. This has been done in the Soft Colour Interaction (SCI) model of ref. [8], where the LEPTO 
[25] event generator was used to estimate higher order effects. Here, starting from the $\mathcal{O}\left(\alpha_{S}\right)$ matrix element, initial-state and final-state parton showers are added to produce multi-parton states. These partons forms strings, which are then hadronized using the string-fragmentation implemented JeTseT. Typically, in the case of the $\gamma^{\star} g \rightarrow q \bar{q}$ process, there are two strings, one connecting the $q$ via radiated gluons to the proton remnant, which is split into two parts, and one connecting the $\bar{q}$ to the remnant in the same way, and a large rapidity gap would be highly unlikely. In SCI model, colour reconnections between the partons are allowed before the hadronization. Any two pairs of colour-connected partons are allowed to reconnect with a given probability $R \approx 0.2$. This is much in the spirit of the model presented in this paper, except that soft gluon exchanges are assumed to be responsible for the reconnections, and that the probability of a given reconnection is somewhat larger than the $1 / N_{c}^{2}$ used in this paper.

There are a number of potential problems with the SCI model. First of all, the initial-state shower implemented in LEPTO is based on conventional Altarelli-Parisi, or DGLAP, evolution [26-29], and there are serious doubts whether such showers are applicable in the case of the small- $x$ processes that make up the bulk of the rapidity-gap events at HERA. Also there are no additional constraints on the colour reconnections, which may lead to highly unlikely string configurations, where a string can go back and forth in rapidity. In addition, since the initial- and final-state showers are completely independent, the model does not include gluon emission diagrams equivalent to the gluon exchange diagram responsible for the reconnections. Finally the approach is very sensitive to where the parton shower is cut off. Nevertheless, choosing a cutoff in virtuality of $4 \mathrm{GeV}^{2}$, a reconnection probability of $R \approx 0.2$, the model can correctly describe the amount of rapidity-gap events and also the distribution in $m_{\mathrm{X}}$ of these events.

In the DCM, in the case of the BGF process, the QCD cascade is described in terms of dipole radiation between two dipoles, one between the $q$ and one part of the proton remnant, and one between the $\bar{q}$ and the other part of the proton remnant. These two dipole-systems are normally treated completely independently. But when the colour-reconnection model presented in this paper is introduced, there could occur some cross-talk. The radiation from a given dipole treats the two diagrams in fig. $6 \mathrm{~b}$ coherently. In the Sudakov form factor used in the generation of emissions, which corresponds to summing up all virtual diagrams of the same order as the emission, the diagram in fig. 6a is therefore in principle included, which could motivate reconnections between the two dipole-systems. However, as an extra hard gluon exchange is very suppressed, reconnections between the systems will only be allowed after the perturbative shower, while reconnections within each system will be allowed earlier.

Disregarding the latter reconnections within the systems, which should not be important for HERA, this model may look very similar to the SCI model. However, there are important differences:

Contrary to the LOR and SCI models, the DCM does include the real emission diagram, 


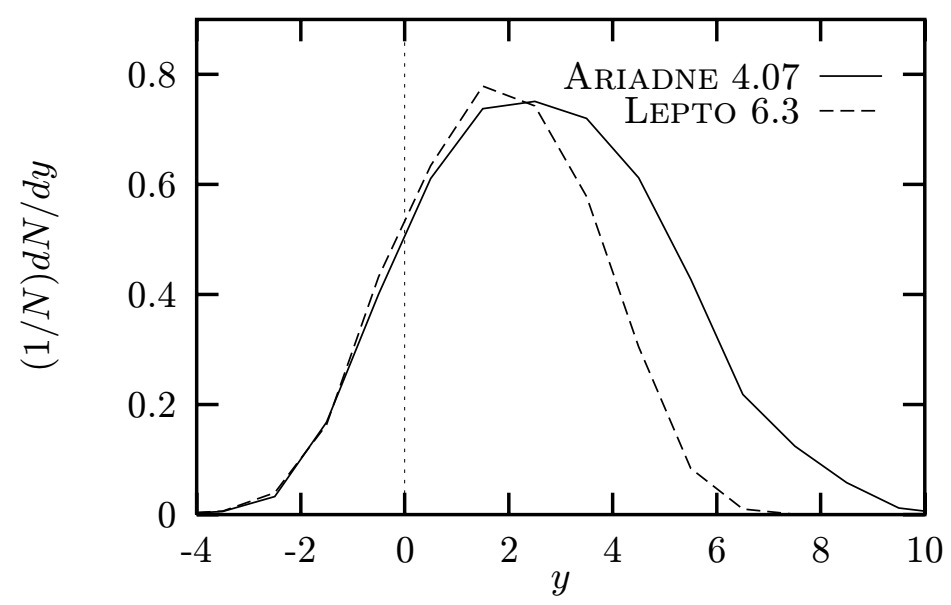

Figure 7: The rapidity distribution of gluons in the lab frame of HERA for ARIADNE (full line) and LEPTO (dashed line).

corresponding to the interference between the diagrams in fig. $6 \mathrm{~b}$, equivalent to the virtual exchange diagram of fig. 6a responsible for the creation of large rapidity gaps.

Also, in the initial-state parton shower used in the SCI, the emissions are strongly ordered both in virtuality $Q^{2}$ and momentum fraction $x$. At HERA, where the bulk of events are at small $Q^{2}$ and $x$, this means that the cascade very quickly "runs out of virtuality", resulting in very little radiation at large rapidities (large $x$ ). In the DCM, however, the cascade is unordered in $x$ (or conversely, if the emissions are traced in increasing $x$, unordered in virtuality), leading to much more perturbative radiation in the forward region, as explained in more detail in ref. [30]. Such an unordered shower is believed to be more appropriate at small $x$, and resembles in this sense the so-called BFKL evolution [31,32]. The effect is very clear in fig. 7 , where the number of gluons per unit of rapidity is shown for LEPTO and Ariadne.

Because of the lack of perturbative radiation in the forward region, the LEPTO program has, in the past, not been able to reproduce the large transverse energy flow in this region measured at HERA $[33,34]$. However, the latest version of LEPTO includes a new nonperturbative treatment of the remnant in the case of BGF processes, which, together with the extra "wrinkling" of the strings introduced by the SCI model as explained above, allows the program to describe the transverse energy flows correctly.

In fig. 8, the result for the rate of rapidity-gap events at HERA is shown for the SCI model, for the colour-reconnected DCM presented here, and for the pomeron-inspired model of ref. [23], which is the default in ARIADNE. It is clear that the DCM gives much fewer events with large rapidity gaps than the SCI model and the default ARIADNE, which both reproduce data fairly well. The reason is, of course, that the DCM radiates more gluons in the forward region, filling up potential gaps. 


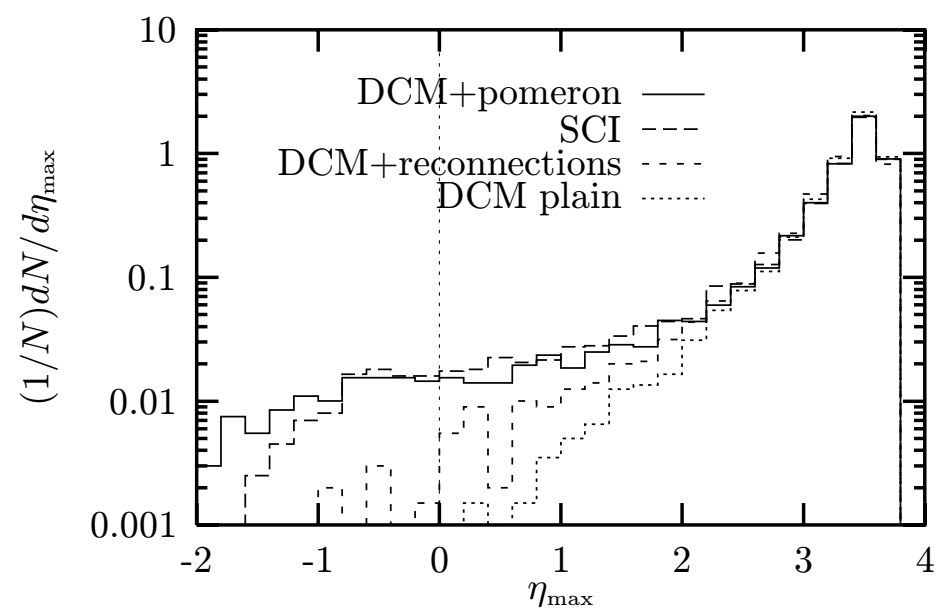

Figure 8: The distribution of $\eta_{\max }$, the pseudo-rapidity of the particle with largest pseudorapidity in the lab frame of HERA. The full line is ARIADNE with the default pomeron inspired model, the long-dashed line is the SCI model as implemented in LEPTO, the shortdashed line is the DCM with colour reconnections and the dotted line is the plain DCM without colour reconnections or pomerons.

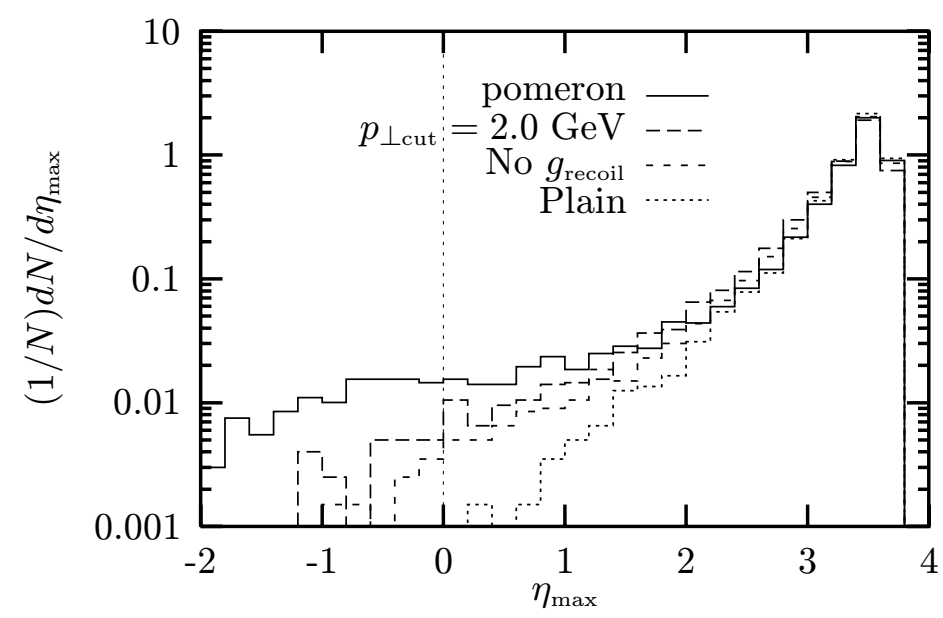

Figure 9: The distribution of $\eta_{\max }$ in the lab frame of HERA. The full line is ARIADNE with the default pomeron inspired model, the long-dashed line is the DCM with colour reconnections and an increased $p_{\perp}$-cutoff, the short-dashed line is the DCM with colour reconnections but without recoil gluons and the dotted line is the plain DCM without colour reconnections or pomerons. 


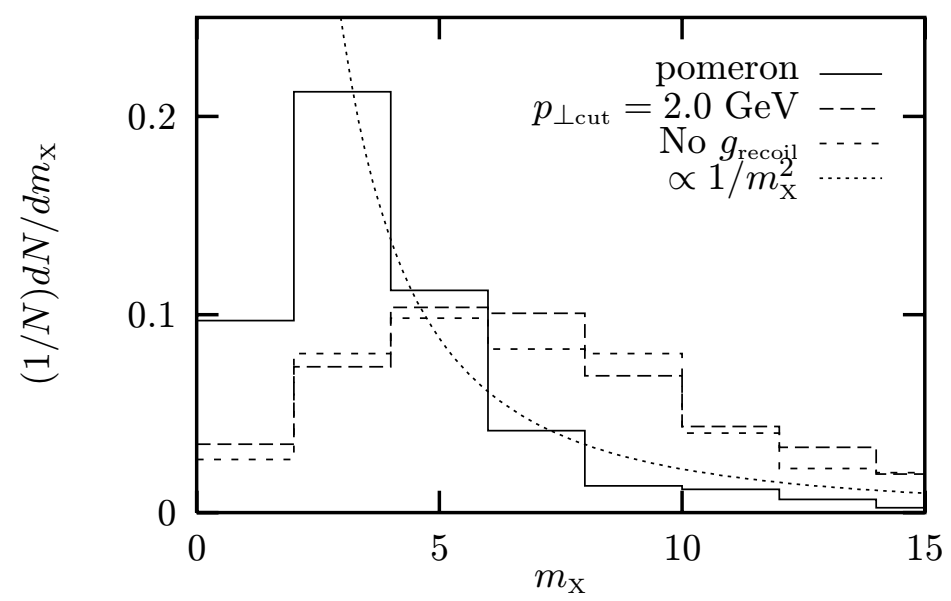

Figure 10: The distribution of $m_{X}$ for events at HERA with $\eta_{\max }<3.2$. The full line is ARIADNE with the default pomeron inspired model, the long-dashed line is the DCM with colour reconnections and an increased $p_{\perp}$-cutoff, the short-dashed line is the DCM with colour reconnections but without recoil gluons and the dotted line is proportional to $1 / m_{X}^{2}$.

In the DCM, just as in the SCI model, the result is dependent on the cutoff in the cascade; indeed, changing the cutoff in transverse momentum from the default $0.6 \mathrm{GeV}$ to $2 \mathrm{GeV}$ reduces the number of gluons emitted, and slightly increases the number of events with large rapidity gaps as in fig. 9. However, examining the distribution in $m_{\mathrm{X}}$ of such events in fig. 10 , the $1 / m_{\mathrm{x}}^{2}$ behaviour found at HERA is not reproduced.

In the DCM, for each gluon emission, there is a possibility of emitting an extra so-called recoil gluon, which absorbs some of the transverse recoil from the truly emitted gluon, as is explained in detail in ref. [13]. These recoil gluons are usually very forward in rapidity, and will decrease the probability of obtaining a large rapidity gap. Although these recoil gluons are natural in the DCM, they are arguably a bit foreign to a normal parton shower scenario. In general, however, they do not affect the behaviour of the cascade very much, and in fig. 9 it is shown that omitting such gluons does not increase the number of rapiditygap events very much and, more important, in fig. 10 it is shown that the $m_{\mathrm{X}}$ distribution is not affected.

So, even if the rate of rapidity-gap events could be reproduced with the colour-reconnected DCM, by increasing abnormally the cutoff in the cascade and/or increasing the reconnection probability above $1 / N_{c}^{2}$, the distribution of perturbative gluons in the DCM is such that the $m_{\mathrm{X}}$ distribution found in such events cannot be described. 


\section{Conclusions}

In all the cases studied in this paper, the effects of the colour-reconnection scenario presented for the Dipole Cascade Model are rather small. This does not imply that such colour reconnections are totally unimportant. At LEP, where the huge amount of statistics allows for very precise measurements of event shapes and particle spectra, the effects of colour reconnection can still be noticeable, although more detailed studies need to be done. For a precise determination of the $W$ mass at LEP 2, the colour-reconnection effects enter as an additional theoretical uncertainty, which may be small compared with the statistical and other systematical errors, but not completely negligible.

At HERA, DIS events with large rapidity gaps have attracted a great amount of attention. This paper has shown that the multitude of such events cannot be explained by colour reconnections within the framework of the DCM. In addition, the DCM is the only QCD cascade model available today, which uses an unordered evolution scenario, which is assumed to be the appropriate way of describing small- $x$ final states, and it was shown that, because of this lack of ordering, the distribution of gluons is such that neither the rate nor the properties of rapidity-gap events can be explained in terms of colour reconnections. However, as long as models based on completely ordered evolutions can be made to reproduce measured data, such as the transverse energy flow, with the help of non-perturbative models, the colour reconnection scenario of ref. [8] cannot be completely ruled out.

The main purpose of this paper was to describe the way colour reconnections are implemented in the ARIADNE program, so that it can be used as a tool for exploiting all kinds

of colour-reconnection phenomena. There are a number of possibly interesting aspects of colour reconnection, which have not been mentioned here. In particular, it is possible to look for direct signals of reconnected events, such as the ones described in ref. [12] for $W^{+} W^{-}$production, but also the search for rapidity gaps at LEP as in ref. [35].

\section{Acknowledgements}

I would like to thank Youri Dokshitzer, Gösta Gustafson and Torbjörn Sjöstrand for valuable discussions.

\section{References}

[1] G. Gustafson, U. Pettersson, P. Zerwas, Phys. Lett. B 209 (1988) 90.

[2] T. Suöstrand, V.A. Khoze, Phys. Rev. Lett. 72 (1994) 28. 
[3] T. Suöstrand, V.A. Khoze, Z. Phys. C 62 (1994) 281.

[4] ZEUS Coll., M. Derrick et Al., Phys. Lett. B 315 (1993) 481.

[5] H1 Coll., I. Abt et Al., Nucl. Phys. B 429 (1994) 477.

[6] L. LÖnnblad, Talk given at the QCD at HERA Workshop, Hamburg, 1994, unpublished.

[7] W. Buchmüller, A. Hebecker, A parton model for diffractive processes in deep inelastic scattering., DESY 95-077 (to appear in Phys. Lett. B), April 1995.

[8] A. Edin, G. Ingelman, J. Rathsman, Rapidity gaps in DIS through soft colour interactions, preprint DESY 95-145, July 1995.

[9] G. Gustafson, Phys. Lett. B 175 (1986) 453.

[10] G. Gustafson, U. Pettersson, Nucl. Phys. B 306 (1988) 746.

[11] L. Lönnblad, Ariadne version 4.07 program and manual, Comput. Phys. Commun. 71 (1992) 15.

[12] G. Gustafson, J. Häkkinen, Z. Phys. C 64 (1994) 659.

[13] B. Andersson et Al., Z. Phys. C 43 (1989) 625.

[14] R. Kleiss, Phys. Lett. B 180 (1986) 400.

[15] B. Andersson, P. Dahlqvist, G. Gustafson, Phys. Lett. B 214 (1988) 604.

[16] B. Andersson, P. Dahlqvist, G. Gustafson, Z. Phys. C 44 (1989) 455.

[17] B Andersson, G. Gustafson, L. Lönnblad, Nucl. Phys. B 339 (1990) 393.

[18] T. Sjöstrand, Comput. Phys. Commun. 82 (1994) 74.

[19] T. SJÖstrand, PYTHIA 5.7 and JETSET 7.4 physics and manual, CERNTH.7112/93, Dec. 1993, (revised Aug. 1994).

[20] G. Marchesini et Al., Comput. Phys. Commun. 67 (1992) 465.

[21] K. Hamacher, M. Weierstall, The next round of hadronic generator tuning heavily based on identified particle data, Wuppertal preprint WU B 95-07, June 1995.

[22] L. LÖnnblad, T. Sjöstrand, Phys. Lett. B 351 (1995) 293.

[23] L. Lönnblad, Z. Phys. C 65 (1995) 285.

[24] G. Ingelman, P. Schlein, Phys. Lett. B 152 (1985) 256.

[25] G. Ingelman, LEPTO version 6.1, in Physics at HERA, edited by W. Buchmüller, G. INGELMAN, volume 3, p. 1366, DESY, 1991. 
[26] V.N. Gribov, L.N. Lipatov, Sov. J. Phys. 15 (1972) 438 and 675.

[27] L.N. Lipatov, Sov. J. Phys. 20 (1975) 94.

[28] G. Altarelli, G. Parisi, Nucl. Phys. B 126 (1977) 298.

[29] Yu.L. Dokshitser, Sov. Phys. JETP 46 (1977) 641.

[30] L. LÖNNBLAD, Small-x effects in $W+$ jets production at the Tevatron, preprint CERNTH/95-212, August 1995.

[31] V.S. Fadin, E.A. Kuraev, L.N. Lipatov, Sov. Phys. JetP 45 (1977) 199.

[32] Ya.Ya. Balitsky, L.N. Lipatov, Sov. J. Nucl. Phys. 28 (1978) 822.

[33] ZEUS Coll., M. Derrick et Al., Z. Phys. C 59 (1993) 231.

[34] H1 Coll., I. Abt et Al., Z. Phys. C 63 (1994) 377.

[35] J. Ellis, D.A. Ross, Search for large rapidity gap events at in $e^{+} e^{-}$annihilation, preprint CERN-TH/95-84, April 1995. 